Pure and Applied Mathematics Quarterly

Volume 7, Number 1

(Special Issue: In honor of

Frederick W. Gehring, Part 1 of 2)

107-120, 2011

\title{
An Entire Function with Simply and Multiply Connected Wandering Domains
}

\author{
Walter Bergweiler \\ Dedicated to Professor Frederick W. Gehring on the occasion of his 80th birthday
}

\begin{abstract}
We modify a construction of Kisaka and Shishikura to show that there exists an entire function $f$ which has both a simply connected and a multiply connected wandering domain. Moreover, these domains are contained in the set $A(f)$ consisting of the points where the iterates of $f$ tend to infinity fast. The results answer questions by Rippon and Stallard.
\end{abstract}

\section{INTRODUCTION AND RESULTS}

Let $f$ be an entire or rational function. The Fatou set $F(f)$ is defined as the set where the iterates $f^{n}$ of $f$ form a normal family. If $U_{0}$ is a component of $F(f)$, then $f^{n}\left(U_{0}\right)$ is contained in a component $U_{n}$ of $F(f)$. If all $U_{n}$ are different, then $U_{0}$ is called a wandering domain of $f$. While a famous theorem of Sullivan [14] says that rational functions do not have wandering domains, it had been shown already earlier by Baker [2] that such domains may exist for transcendental entire functions. While the wandering domain in Baker's example was multiply connected, examples of simply connected wandering domains were given later by various authors; see $[9$, p. 106], [14, p. 414], [3, p. 564, p. 567]

Received December 14, 2006.

1991 Mathematics Subject Classification. Primary 37F10; Secondary 30D05, 30C62.

Supported by the G.I.F., the German-Israeli Foundation for Scientific Research and Development, Grant G-809-234.6/2003 
and [7, p. 222]. Baker [4, Theorem 2] showed that his construction can be modified to yield wandering domains of infinite connectivity. Recently Kisaka and Shishikura [11] constructed an example with a multiply connected wandering domain of finite connectivity, thereby answering a question of Baker. In fact, they showed that the connectivity may take any preassigned value. Here we modify the construction of Kisaka and Shishikura to prove the following result.

Theorem 1. There exists an entire function which has both a simply connected and a multiply connected wandering domain.

The question whether an entire function with this property exists had been raised by Rippon and Stallard [13, p. 1125, Remark 3]. In the same paper, Rippon and Stallard also asked a question about the set $A(f)$ introduced in [6]. This is defined by

$$
A(f):=\left\{z: \text { there exists } L \in \mathbb{N} \text { such that }\left|f^{n}(z)\right|>M\left(R, f^{n-L}\right) \text { for } n>L\right\},
$$

where $M(r, f):=\max _{|z|=r}|f(z)|$ and $R>\min _{z \in J(f)}|z|$. Roughly speaking, $A(f)$ consists of the points $z$ where $f^{n}(z)$ tends to infinity "as fast as possible." Rippon and Stallard showed that $A(f)$ has no bounded components and that the closure of every multiply connected wandering domain is contained in $A(f)$. They also showed that if a simply connected wandering domain intersects $A(f)$, then it must lie entirely in $A(f)$, and they ask [13, p. 1126, Remark 4] whether an entire function $f$ with such a simply connected wandering domain exists. It turns out that an example with this property is provided by the function constructed in Theorem 1.

Theorem 2. There exists an entire function $f$ for which $A(f)$ contains a simply connected wandering domain.

As mentioned, our construction is largely based on that of Kisaka and Shishikura. We state two of their lemmas in $\S 2$ and then repeat their construction in $\S 3$. There is only one minor change in the construction, which will be explained at the beginning of $\S 4$. In the remainder of $\S 4$ we then show that the function constructed has a simply connected wandering domain, thereby proving Theorem 1 . In $\S 5$ we prove Theorem 2 .

Acknowledgment. I would like to thank the referee for a careful reading of the manuscript and useful suggestions. 


\section{Two lemmas of Kisaka And Shishikura}

Kisaka and Shishikura first construct a quasiregular map $g: \mathbb{C} \rightarrow \mathbb{C}$ and then obtain the entire function $f$ with the following lemma.

Lemma 1. [11, Theorem 3.1] Let $g$ be a quasiregular mapping from $\mathbb{C}$ to $\mathbb{C}$. Suppose that there are (disjoint) measurable sets $E_{j} \subset \mathbb{C}(j=1,2, \ldots)$ satisfying:

(a) For almost every $z \in \mathbb{C}$, the $g$-orbit of $z$ passes $E_{j}$ at most once for every $j$;

(b) $g$ is $K_{j}$-quasiregular on $E_{j}$;

(c) $K_{\infty}:=\prod_{j=1}^{\infty} K_{j}<\infty$;

(d) $g$ is holomorphic a.e. outside $\bigcup_{j=1}^{\infty} E_{j}$ (i.e. $\frac{\partial g}{\partial \bar{z}}=0$ a.e. on $\mathbb{C} \backslash \bigcup_{j=1}^{\infty} E_{j}$ ).

Then there exists a $K_{\infty}$-quasiconformal map $\varphi$ such that $f=\varphi \circ g \circ \varphi^{-1}$ is an entire function

In order to construct $g$ they need to "interpolate" two polynomials given on circles by a quasiregular map with small dilatation. This is done with the following result, where log denotes the principal branch of the logarithm.

Lemma 2. [11, Lemma 6.3] Let $k \in \mathbb{N}, b, \omega \in \mathbb{C} \backslash\{0\}$ and $\rho^{\sharp}, \lambda^{\sharp}, \rho^{b}, \lambda^{b} \in \mathbb{R}$ with $0<\lambda^{b}<\rho^{b}<1<\rho^{\sharp}<\lambda^{\sharp}$.

(a) Suppose that these constants satisfy

$\rho^{\sharp} \geq 2|\omega|, \quad \lambda^{\sharp} \geq e \rho^{\sharp}, \quad C^{\sharp}:=1-\frac{1}{k+1}\left(\frac{|\log b|}{\log \left(\lambda^{\sharp} / \rho^{\sharp}\right)}+\frac{4|\omega|}{\rho^{\sharp}}\right)>0$,

Then the $\operatorname{map} b z^{k}(z-\omega)$ on $|z|=\rho^{\sharp}$ and $z^{k+1}$ on $|z|=\lambda^{\sharp}$ can be interpolated on $\rho^{\sharp} \leq|z| \leq \lambda^{\sharp}$ with a $K$-quasiregular map $g$ where $K \leq 1 / C^{\sharp}$.

(b) Suppose that these constants satisfy

$$
|\omega| \geq 2 \rho^{b}, \quad \rho^{b} \geq e \lambda^{b}, \quad C^{b}:=1-\frac{1}{k}\left(\frac{|\log (-b \omega)|}{\log \left(\rho^{b} / \lambda^{b}\right)}+\frac{4 \rho^{b}}{|\omega|}\right)>0 .
$$

Then the map $b z^{k}(z-\omega)$ on $|z|=\rho^{b}$ and $z^{k}$ on $|z|=\lambda^{b}$ can be interpolated on $\lambda^{b} \leq|z| \leq \rho^{b}$ with a $K$-quasiregular map $g$ where $K \leq 1 / C^{b}$. 


\section{Construction of $f$}

As mentioned, we follow closely the ideas of Kisaka and Shishikura and will first construct a quasiregular map $g: \mathbb{C} \rightarrow \mathbb{C}$ and then obtain $f$ via Lemma 1 .

We denote by $\operatorname{ann}(r, R)$ the open annulus with inner radius $r$ and outer radius $R$; that is, $\operatorname{ann}(r, R):=\{z \in \mathbb{C}: r<|z|<R\}$. The idea is to choose sequences $\left(a_{n}\right)$ and $\left(R_{n}\right)$ such that the map $z \mapsto a_{n} z^{n+1} \operatorname{maps} \operatorname{ann}\left(R_{n}, R_{n+1}\right)$ onto $\operatorname{ann}\left(R_{n+1}, R_{n+2}\right)$. The map $g$ will then be defined by $g(z)=a_{n} z^{n+1}$ on a large subannulus of $\operatorname{ann}\left(R_{n}, R_{n+1}\right)$, and will interpolate the mappings $z \mapsto a_{n-1} z^{n}$ and $z \mapsto a_{n} z^{n+1}$ in an annulus containing the circle $\left\{z:|z|=R_{n}\right\}$.

Choosing $R_{1}>R_{0}:=1$ we obtain sequences $\left(R_{n}\right)$ and $\left(a_{n}\right)$ as required by putting

$$
R_{n+1}:=\frac{R_{n}^{n+1}}{R_{n-1}^{n}}
$$

and

$$
a_{n}:=\frac{R_{n+1}}{R_{n}^{n+1}}=\frac{1}{R_{n-1}^{n}} .
$$

Various estimates in the sequel will require that $R_{1}$ has been chosen large enough. Note that with $\gamma:=\log R_{1}$ we have

$$
\log \frac{R_{n+1}}{R_{n}}=n \log \frac{R_{n}}{R_{n-1}}=\ldots=n ! \log \frac{R_{1}}{R_{0}}=\gamma n ! .
$$

We define sequences $\left(P_{n}\right),\left(Q_{n}\right),\left(S_{n}\right)$ and $\left(T_{n}\right)$ by

$$
\log \frac{T_{n}}{S_{n}}=\log \frac{S_{n}}{R_{n}}=\log \frac{R_{n}}{Q_{n}}=\log \frac{Q_{n}}{P_{n}}=\sqrt{\log \frac{R_{n+1}}{R_{n}}}=\sqrt{\gamma n !}
$$

Choosing $R_{1}>e$ we have $\gamma>1$ and thus

$$
\frac{T_{n}}{S_{n}}=\frac{S_{n}}{R_{n}}=\frac{R_{n}}{Q_{n}}=\frac{Q_{n}}{P_{n}}>e .
$$

We also have

$$
\begin{aligned}
\log \frac{P_{n+1}}{T_{n}} & =-\log \frac{Q_{n+1}}{P_{n+1}}-\log \frac{R_{n+1}}{Q_{n+1}}+\log \frac{R_{n+1}}{R_{n}}-\log \frac{S_{n}}{R_{n}}-\log \frac{T_{n}}{S_{n}} \\
& =-2 \sqrt{\gamma(n+1) !}+\gamma n !-2 \sqrt{\gamma n !} \\
& >0
\end{aligned}
$$


for all $n \in \mathbb{N}$, provided that $R_{1}$ and hence $\gamma$ is large enough. Thus

$$
P_{n}<Q_{n}<R_{n}<S_{n}<T_{n}<P_{n+1}
$$

for all $n \in \mathbb{N}$. We now define

$$
b_{n}:=-\frac{(n+1)^{2}}{n+2}\left(\frac{n+1}{n}\right)^{n} a_{n}=-\frac{(n+1)^{2}}{n+2}\left(\frac{n+1}{n}\right)^{n} \frac{R_{n+1}}{R_{n}^{n+1}}
$$

for $n \in \mathbb{N}$. We also put $E_{1}:=\operatorname{ann}\left(P_{2}, Q_{2}\right)$ and

$$
E_{n}:=\operatorname{ann}\left(S_{n}, T_{n}\right) \cup \operatorname{ann}\left(P_{n+1}, Q_{n+1}\right)
$$

for $n \geq 2$.

We shall show that there exists a quasiregular map $g: \mathbb{C} \rightarrow \mathbb{C}$ with the following properties:

(i) $g(z)=a_{1} z^{2}$ for $|z| \leq P_{2}$;

(ii) $g(z)=a_{n} z^{n+1}$ for $T_{n} \leq|z| \leq P_{n+1}$ and $n \geq 2$;

(iii) $g(z)=b_{n}\left(z-R_{n}\right) z^{n}$ for $Q_{n} \leq|z| \leq S_{n}$ and $n \geq 2$;

(iv) $g$ is $K_{n}$-quasiregular in $E_{n}$ for $n \geq 1$, with $K_{n}:=1+1 / n^{2}$;

(v) $g\left(\operatorname{ann}\left(S_{n}, Q_{n+1}\right)\right) \subset \operatorname{ann}\left(S_{n+1}, Q_{n+2}\right)$ for $n \geq 1$.

Since $E_{n} \subset \operatorname{ann}\left(S_{n}, Q_{n+1}\right)$ and since the annuli $\operatorname{ann}\left(S_{n}, Q_{n+1}\right)$ are pairwise disjoint it then follows that $g$ satisfies the hypothesis of Lemma 1. Thus there exists a quasiconformal map $\varphi$ such that $f:=\varphi \circ g \circ \varphi^{-1}$ is entire. This function $f$ then has the desired properties.

In order to show that a map $g$ with the properties stated exists, we simply define $g$ by (i), (ii), (iii) in the ranges given there and thus have defined $g$ in $\mathbb{C} \backslash \bigcup_{n=1}^{\infty} E_{n}$.

To define $g$ in $\operatorname{ann}\left(P_{n}, Q_{n}\right)$, where $n \geq 2$, we consider $G(z):=g\left(R_{n} z\right) / R_{n+1}$. For $|z|=\lambda^{b}:=P_{n} / R_{n}$ we then have

$$
G(z)=a_{n-1} \frac{\left(R_{n} z\right)^{n}}{R_{n+1}}=\frac{R_{n}}{R_{n-1}^{n}} \frac{R_{n}^{n} z^{n}}{R_{n+1}}=z^{n}
$$

and for $|z|=\varrho^{b}:=Q_{n} / R_{n}$ we have

$$
G(z)=b_{n} \frac{\left(R_{n} z-R_{n}\right)\left(R_{n} z\right)^{n}}{R_{n+1}}=b_{n} \frac{R_{n}^{n+1}}{R_{n+1}}(z-1) z^{n}=c_{n}(z-1) z^{n}
$$

with

$$
c_{n}:=\frac{b_{n} R_{n}^{n+1}}{R_{n+1}}=-\frac{(n+1)^{2}}{n+2}\left(\frac{n+1}{n}\right)^{n} .
$$


Now

$$
\varrho^{b}=\frac{Q_{n}}{R_{n}}=\exp (-\sqrt{\gamma n !})
$$

and

$$
\lambda^{b}=\frac{P_{n}}{R_{n}}=\exp (-2 \sqrt{\gamma n !})=\left(\varrho^{b}\right)^{2} .
$$

Thus $\varrho^{b} \geq e \lambda^{b}$ since $\gamma \geq 1$ and also $2 \varrho^{b} \leq 1$. By Lemma 2 , (b), there exists a $K$-quasiregular map $G_{n}:\left\{z \in \mathbb{C}: \lambda^{b} \leq|z| \leq \varrho^{b}\right\} \rightarrow \mathbb{C}$ such that $G_{n}(z)=z^{n}$ for $|z|=\lambda^{b}$ and $G_{n}(z)=c_{n}(z-1) z^{n}$ for $|z|=\varrho^{b}$, with $K \leq 1 / C^{b}$ where

$$
C^{b}:=1-\frac{1}{n}\left(\frac{\left|\log \left(-c_{n}\right)\right|}{\log \left(\varrho^{b} / \lambda^{b}\right)}+4 \varrho^{b}\right)
$$

provided that $C^{b}>0$. But since

$$
\left|\log \left(-c_{n}\right)\right|=\log \left(\frac{(n+1)^{2}}{n+2}\left(\frac{n+1}{n}\right)^{n}\right) \leq \log ((n+1) e)=1+\log (n+1)
$$

we may in fact achieve that

$$
C^{b} \geq 1-\frac{1}{n}\left(\frac{1+\log (n+1)}{\sqrt{\gamma n !}}+4 \exp (-\sqrt{\gamma n !})\right) \geq 1-\frac{1}{(n-1)^{2}+1}
$$

for all $n \geq 2$ by choosing $\gamma$ large enough. Thus

$$
K \leq \frac{1}{C^{b}} \leq 1+\frac{1}{(n-1)^{2}}
$$

Putting

$$
g(z):=R_{n+1} G_{n}\left(\frac{z}{R_{n}}\right)
$$

for $z \in \operatorname{ann}\left(P_{n}, Q_{n}\right) \subset E_{n-1}$ we see that (iv) holds for $z \in E_{n-1} \cap \operatorname{ann}\left(P_{n+1}, Q_{n+1}\right)$. Similarly we define $g$ in the remaining part of $E_{n}$; that is, in $E_{n} \cap \operatorname{ann}\left(S_{n}, T_{n}\right)$. Here we use the first part of Lemma 2. 
To prove (v) we note that if $z \in \operatorname{ann}\left(S_{n}, Q_{n+1}\right)$, then, by the maximum principle,

$$
\begin{aligned}
|g(z)| & \leq \max _{|\zeta|=Q_{n+1}}|g(\zeta)| \\
& =\max _{|\zeta|=Q_{n+1}}\left|b_{n+1}\left(\zeta-R_{n+1}\right) \zeta^{n+1}\right| \\
& =\left|b_{n+1}\right|\left(R_{n+1}+Q_{n+1}\right) Q_{n+1}^{n+1} \\
& =\frac{(n+2)^{2}}{n+3}\left(\frac{n+2}{n+1}\right)^{n+1} R_{n+2}\left(1+\frac{Q_{n+1}}{R_{n+1}}\right)\left(\frac{Q_{n+1}}{R_{n+1}}\right)^{n+1} \\
& \leq 2 e(n+2) R_{n+2}\left(\frac{Q_{n+1}}{R_{n+1}}\right)^{n+1} \\
& =2 e(n+2) R_{n+2} \exp (-(n+1) \sqrt{\gamma(n+1) !}) \\
& \leq R_{n+2} \exp (-\sqrt{\gamma(n+2) !}) \\
& =Q_{n+2}
\end{aligned}
$$

if $\gamma$ is large enough. Similarly, noting that $g$ has no zeros in $\operatorname{ann}\left(S_{n}, Q_{n+1}\right)$ and using the minimum principle, we find that $|g(z)| \geq S_{n+1}$ for $z \in \operatorname{ann}\left(S_{n}, Q_{n+1}\right)$. We deduce that (v) holds.

As in the paper of Kisaka and Shishikura we deduce from (v) that $g^{n}(z) \rightarrow$ $\infty$ as $n \rightarrow \infty$ for $z \in \operatorname{ann}\left(S_{1}, Q_{2}\right)$, while $g(0)=0$ by (i). This implies that $\varphi\left(\operatorname{ann}\left(S_{1}, Q_{2}\right)\right)$ lies in a multiply connected component $U_{1}$ of the Fatou set of $f$, with $\left.f^{n}\right|_{U_{1}} \rightarrow \infty$ as $n \rightarrow \infty$. Since multiply connected components of the Fatou set are always bounded by a result of Baker [1, Theorem 1], this implies that $f$ has a multiply connected wandering domain.

Remark. The Fatou set and the other concepts of complex dynamics can also be defined for quasiregular maps, by carrying over the definitions from the holomorphic case literally. In order to retain the basic features of the theory one has to require, however, that all iterates of $g$ are $K$-quasiregular with the same $K$. Such maps are called uniformly quasiregular. That our function $g$ is uniformly quasiregular follows directly from the definition of $g$, or trivially from the representation $g=\varphi^{-1} \circ f \circ \varphi$. Lemma 1 says, essentially, that uniformly quasiregular selfmaps of the plane are quasiconformally conjugated to entire functions; see also $[8,10]$ for this result. 
4. Proof of Theorem 1: $f$ has a Simply ConneCted WANDERING Domain

The sequence $\left(\xi_{n}\right)$ of critical points of $g$ is given by $\xi_{1}:=0$ and $\xi_{n}:=\frac{n}{n+1} R_{n}$. The only difference between the present construction and that of Kisaka and Shishikura concerns the orbits of these points. While we have chosen the values $b_{n}$ such that

$$
g\left(\xi_{n}\right)=b_{n}\left(\xi_{n}-R_{n}\right) \xi_{n}^{n}=-b_{n} \frac{R_{n}}{n+1}\left(\frac{n}{n+1}\right)^{n} R_{n}^{n}=\frac{n+1}{n+2} R_{n+1}=\xi_{n+1}
$$

for $n \geq 2$, Kisaka and Shishikura worked with different values of $b_{n}$ which yielded $g\left(\xi_{n}\right)=R_{n+1}$ and hence $g^{2}\left(\xi_{n}\right)=0$.

Denote by $D(a, r)$ the disk of radius $r$ around $a$. Let $\delta>0$ and define $D_{n}:=$ $D\left(\xi_{n}, \delta R_{n} / n^{4}\right)$ for $n \geq 2$. We shall show that if $\delta$ is sufficiently small, then $g\left(D_{n}\right) \subset D_{n+1}$ for all $n$. This implies that $D_{n} \subset F(g)$ for $n \geq 2$. We will then show that $D_{n}$ lies in a simply connected wandering domain of $g$ and thus $\varphi\left(D_{n}\right)$ lies in a simply connected wandering domain $V_{n}$ of $f$. Moreover, we will see in $\S 5$ that $V_{n} \subset A(f)$ for all $n$.

First we note that if $\delta$ is small enough, then $D_{n} \subset \operatorname{ann}\left(Q_{n}, R_{n}\right)$ so that $g$ is holomorphic in $D_{n}$ and

$$
\begin{aligned}
\left|g^{\prime \prime}(z)\right| & =\left|b_{n}\left(n(n+1) z^{n-1}-R_{n} n(n-1) z^{n-2}\right)\right| \\
& \leq\left|b_{n}\right|(n(n+1)+n(n-1)) R_{n}^{n-1} \\
& =2 n^{2}\left|b_{n}\right| R_{n}^{n-1}
\end{aligned}
$$

for $z \in D_{n}$. Thus

$$
\begin{aligned}
\left|g^{\prime}(z)\right| & =\left|g^{\prime}(z)-g^{\prime}\left(\xi_{n}\right)\right| \\
& \leq \int_{\xi_{n}}^{z}\left|g^{\prime \prime}(\zeta)\right||d \zeta| \\
& \leq 2 n^{2}\left|b_{n}\right| R_{n}^{n-1} \frac{\delta R_{n}}{n^{4}} \\
& =\frac{2 \delta}{n^{2}}\left|b_{n}\right| R_{n}^{n}
\end{aligned}
$$


for $z \in D_{n}$. It follows that if $z \in D_{n}$, then

$$
\begin{aligned}
\left|g(z)-\xi_{n+1}\right| & =\left|g(z)-g\left(\xi_{n}\right)\right| \\
& \leq \int_{\xi_{n}}^{z}\left|g^{\prime}(\zeta)\right||d \zeta| \\
& \leq \frac{2 \delta}{n^{2}}\left|b_{n}\right| R_{n}^{n} \frac{\delta R_{n}}{n^{4}} \\
& =\frac{2 \delta^{2}}{n^{6}} \frac{(n+1)^{2}}{n+2}\left(\frac{n+1}{n}\right)^{n} R_{n+1} \\
& \leq \frac{2 \delta^{2} e(n+1)}{n^{6}} R_{n+1} \\
& \leq \frac{\delta R_{n+1}}{(n+1)^{4}},
\end{aligned}
$$

provided $\delta$ is sufficiently small. Thus $g\left(D_{n}\right) \subset D_{n+1}$ for $n \geq 2$. As already mentioned, this implies that $D_{n}$ lies in a Fatou component $V_{n}^{\prime}$ of $g$ and thus $\varphi\left(D_{n}\right)$ lies in the Fatou component $V_{n}:=\varphi\left(V_{n}^{\prime}\right)$ of $f$. By $U_{n}^{\prime}$ we denote the multiply connected Fatou component of $g$ which contains $\operatorname{ann}\left(S_{n}, Q_{n+1}\right)$, and by $U_{n}:=\varphi\left(U_{n}^{\prime}\right)$ the corresponding Fatou component of $f$. As mentioned at the end of $\S 3$, the $U_{n}$ are wandering domains. In fact, we have $U_{m} \neq U_{l}$ for $m \neq l$ (and thus $U_{m}^{\prime} \neq U_{l}^{\prime}$ for $m \neq l$ ). We shall show that $U_{m}^{\prime} \neq V_{l}^{\prime}$ (and thus $U_{m} \neq V_{l}$ ) for all $m$ and $l$. Since $V_{l}^{\prime}$ lies "between" the annuli $\operatorname{ann}\left(S_{l-1}, Q_{l}\right)$ and $\operatorname{ann}\left(S_{l}, Q_{l+1}\right)$ and thus "between" the domains $U_{l-1}^{\prime}$ and $U_{l}^{\prime}$, it suffices to show that $U_{m}^{\prime} \neq V_{l}^{\prime}$ for $l=m$ and $l=m+1$.

Suppose that $U_{m}^{\prime}=V_{l}^{\prime}$ where $l=m$ or $l=m+1$. Since $D_{l} \subset V_{l}^{\prime}$ and $\operatorname{ann}\left(T_{m}, P_{m+1}\right) \subset U_{m}^{\prime}$ there exists a simply connected domain $\Omega_{m}$ with

$$
D_{l} \cup \operatorname{ann}\left(T_{m}, P_{m+1}\right) \backslash\left(-P_{m+1}, T_{m}\right) \subset \Omega_{m} \subset U_{m}^{\prime} .
$$

Since $g^{n}(z) \neq 0$ for $z \in \Omega_{m}$ and since the $g^{n}$ are $K$-quasiregular for some $K$, we may define for $n>m$ a $K$-quasiregular map $h_{n}: \Omega_{m} \rightarrow \mathbb{C}$ by

$$
h_{n}(z)=\left(\frac{g^{n-m}(z)}{R_{n}}\right)^{m ! / n !},
$$

for some branch of the root. We will show that the $h_{n}$ form a normal family so that $\left(h_{n}\right)$ has a convergent subsequence, say $h_{n_{k}} \rightarrow h$. Next we will show that $h_{n}(z)=h(z)=z / R_{m}$ for $z \in \operatorname{ann}\left(T_{m}, P_{m+1}\right) \backslash\left(-P_{m+1}, T_{m}\right)$, if the branch of the root has been suitably chosen. This implies in particular that $h$ is nonconstant. 
On the other hand, we will see that $h$ is constant in $D_{l}$ so that we obtain a contradiction.

To prove that $\left(h_{n}\right)$ is normal we note that if $z \in \Omega_{m} \subset U_{m}^{\prime}$, then $g^{n-m}(z) \in U_{n}^{\prime}$ and thus $\left|g^{n-m}(z)\right| \leq S_{n+1}$, since $\operatorname{ann}\left(S_{n+1}, Q_{n+2}\right) \subset U_{n+1}^{\prime}$. Hence

$$
\left|h_{n}(z)\right| \leq\left|\frac{S_{n+1}}{R_{n}}\right|^{m ! / n !}=\left|\frac{S_{n+1}}{R_{n+1}} \frac{R_{n+1}}{R_{n}}\right|^{m ! / n !}
$$

for $z \in \Omega_{m}$. We deduce that

$$
\begin{aligned}
\log \left|h_{n}(z)\right| & \leq \frac{m !}{n !}\left(\log \frac{S_{n+1}}{R_{n+1}}+\log \frac{R_{n+1}}{R_{n}}\right) \\
& =\frac{m !}{n !}(\sqrt{\gamma(n+1) !}+\gamma n !) \\
& \leq 2 \gamma m !
\end{aligned}
$$

for $z \in \Omega_{m}$ and large $n$, and this yields the desired normality.

It is not difficult to see by induction that if $z \in \operatorname{ann}\left(T_{m}, P_{m+1}\right) \backslash\left(-P_{m+1}, T_{m}\right)$ and $n>m$, then

$$
g^{n-m}(z)=R_{n}\left(\frac{z}{R_{m}}\right)^{n ! / m !} \in \operatorname{ann}\left(T_{n}, P_{n+1}\right) \backslash\left(-P_{n+1}, T_{n}\right)
$$

so that

$$
h_{n}(z)=h(z)=\frac{z}{R_{m}}
$$

if the branch of the root in the definition of $h_{n}$ has been suitable chosen. In particular, $h$ is nonconstant.

For $z \in D_{l}$ we have

$$
g^{n-m}(z) \in D_{n-m+l}
$$

and thus $g^{n-m}(z) \in D_{n}$ or $g^{n-m}(z) \in D_{n+1}$ depending on whether $l=m$ or $l=m+1$. In the first case we have

$$
\frac{g^{n-m}(z)}{R_{n}} \in D\left(\frac{\xi_{n}}{R_{n}}, \frac{\delta}{n^{4}}\right)=D\left(\frac{n}{n+1}, \frac{\delta}{n^{4}}\right) \subset D\left(1, \frac{1}{2}\right)
$$

for large $n$, and this implies that $h$ is constant in $D_{l}$. In the second case, that is for $l=m+1$, we have

$$
\frac{g^{n-m}(z)}{R_{n}} \in D\left(\frac{\xi_{n+1}}{R_{n}}, \frac{\delta R_{n+1}}{(n+1)^{4} R_{n}}\right)
$$


and hence

$$
\left(1-\frac{2}{n}\right) \frac{R_{n+1}}{R_{n}} \leq\left|\frac{g^{n-m}(z)}{R_{n}}\right| \leq \frac{R_{n+1}}{R_{n}}
$$

for large $n$. Since $\sqrt[n !]{1-2 / n} \rightarrow 1$ as $n \rightarrow \infty$ and

$$
\left|\frac{R_{n+1}}{R_{n}}\right|^{m ! / n !}=\exp (\gamma m !)
$$

we deduce that $|h(z)|=\exp (\gamma m !)$ for $z \in D_{l}$. Thus $h$ is constant in $D_{l}$ in this case as well. As already noted, this is a contradiction. This completes the proof that $U_{m}^{\prime} \neq V_{l}^{\prime}$ and hence $U_{m} \neq V_{l}$ for all $l$ and $m$.

It remains to show that $V_{l}$ is simply connected for all $l$. Suppose that this is not the case. It is not difficult to show that then all $V_{l}$ are multiply connected. By a result of Baker [3, Theorem 3.1] there exists $k \in \mathbb{N}$ and a Jordan curve $\tau$ in $V_{k}$ whose interior contains 0 . This implies that $D\left(0, Q_{k}\right)$ is contained in the interior of $\tau^{\prime}:=\varphi^{-1}(\tau)$. The argument principle implies that the winding number of $g\left(\tau^{\prime}\right)$ around 0 is at least $k$. This winding number is equal to the winding number of $f(\tau)$ around 0 , and thus the latter winding number is also at least $k$. Induction shows that the winding number of $\tau_{n}:=f^{n-k}(\tau)$ around 0 is at least $(n-1) ! /(k-1)$ ! for $n \geq k$. A contradiction will now be obtained from a consideration of the hyperbolic length of $\tau_{n}$ in $V_{n}$. We denote the hyperbolic length of a curve $\sigma$ in a hyperbolic domain $U$ by $\ell(\sigma, U)$. By the Schwarz-PickLemma we have

$$
\ell\left(\tau_{n}, V_{n}\right) \leq \ell\left(\tau, V_{k}\right)
$$

for all $n \geq k$. On the other hand, we have $V_{n}^{\prime} \subset \operatorname{ann}\left(Q_{n}, S_{n}\right)$ and thus $V_{n} \subset$ $\varphi\left(\operatorname{ann}\left(Q_{n}, S_{n}\right)\right)$. This implies that

$$
\ell\left(\tau_{n}, V_{n}\right) \geq \ell\left(\tau_{n}, \varphi\left(\operatorname{ann}\left(Q_{n}, S_{n}\right)\right)\right) .
$$

Now $\operatorname{ann}\left(Q_{n}, S_{n}\right)$ is an annulus of modulus $\log \left(S_{n} / Q_{n}\right) /(2 \pi)$. Since $\varphi$ is $K$-quasiconformal this yields that $\varphi\left(\operatorname{ann}\left(Q_{n}, S_{n}\right)\right)$ has modulus at most

$$
\frac{K}{2 \pi} \log \left(\frac{S_{n}}{Q_{n}}\right)=\frac{1}{2 \pi} \log \left(\left(\frac{S_{n}}{Q_{n}}\right)^{K}\right) .
$$

It follows that there exists a conformal map $\psi: \varphi\left(\operatorname{ann}\left(Q_{n}, S_{n}\right)\right) \rightarrow \operatorname{ann}\left(1, r_{n}\right)$ where $r_{n} \leq\left(S_{n} / Q_{n}\right)^{K}$. We may choose $\psi$ such that $|\psi(\varphi(z))| \rightarrow 1$ as $|z| \rightarrow Q_{n}$. Put $\sigma_{n}:=\psi\left(\tau_{n}\right)$. Then

$$
\ell\left(\sigma_{n}, \operatorname{ann}\left(1, r_{n}\right)\right)=\ell\left(\tau_{n}, \varphi\left(\operatorname{ann}\left(Q_{n}, S_{n}\right)\right)\right)
$$


and the winding number of $\sigma_{n}$ around 0 is the same as that of $\tau_{n}$ and thus at least $(n-1) ! /(k-1)$ !. We note that the density $\varrho(z)$ of the hyperbolic metric in $\operatorname{ann}\left(1, r_{n}\right)$ is given by (see, e. g., $[12$, p. 12])

$$
\varrho(z)=\frac{\pi}{|z| \sin \left(\pi \log |z| / \log r_{n}\right) \log r_{n}} .
$$

In particular we have $\varrho(z) \geq \pi /\left(|z| \log r_{n}\right)$ and thus we conclude that

$$
\ell\left(\sigma_{n}, \operatorname{ann}\left(1, r_{n}\right)\right)=\int_{\sigma_{n}} \varrho(w)|d w| \geq \frac{\pi}{\log r_{n}} \int_{\sigma_{n}} \frac{|d w|}{|w|} \geq \frac{2 \pi^{2}}{\log r_{n}} \frac{(n-1) !}{(k-1) !} .
$$

Since $\log r_{n} \leq K \log \left(S_{n} / Q_{n}\right)=2 K \sqrt{\gamma n !}$ we deduce that

$$
\ell\left(\sigma_{n}, \operatorname{ann}\left(1, r_{n}\right)\right) \geq \frac{\pi^{2}}{K(k-1) !} \frac{(n-1) !}{\sqrt{\gamma n !}}
$$

so that

$$
\ell\left(\sigma_{n}, \operatorname{ann}\left(1, r_{n}\right)\right) \rightarrow \infty
$$

as $n \rightarrow \infty$.

On the other hand, our previous estimates imply that

$$
\ell\left(\sigma_{n}, \operatorname{ann}\left(1, r_{n}\right)\right)=\ell\left(\tau_{n}, \varphi\left(\operatorname{ann}\left(Q_{n}, S_{n}\right)\right)\right) \leq \ell\left(\tau_{n}, V_{n}\right) \leq \ell\left(\tau, V_{k}\right) .
$$

This is a contradiction. Thus $V_{\ell}$ is simply connected for all $\ell$. This completes the proof of Theorem 1.

Remark. Except for the fixed point $\varphi(0)$, the critical points of $f$ are contained in the simply connected wandering domains $V_{n}$ of $f$. Thus the wandering domains $U_{n}$ do not contain critical points. Using this it can be shown with the arguments of Kisaka and Shishikura (in particular, [11, Proposition 4.5]) that the $U_{n}$ are doubly connected.

\section{Proof of Theorem 2: the $V_{k}$ ARe in $A(f)$}

We will use the following characterisation of the set $A(f)$ given by Rippon and Stallard [13, Lemma 2.4]. Here we denote for a domain $U$ by $\tilde{U}$ the union of $U$ and its bounded complementary components.

Lemma 3. Let $f$ be a transcendental entire function and let $D$ be a domain intersecting the Julia set of $f$. Then

$A(f)=\left\{z:\right.$ there exists $L \in \mathbb{N}$ such that $f^{n+L}(z) \notin \widetilde{f^{n}(D)}$ for $\left.n \in \mathbb{N}\right\}$. 
We apply this result with $D:=U_{1}$. It follows from the maximum principle that $\widetilde{f^{n}\left(U_{1}\right)}=\widetilde{U_{n+1}}$. Since $U_{m}^{\prime} \subset D\left(0, S_{m+1}\right)$ we have $V_{l}^{\prime} \cap \widetilde{U_{m}^{\prime}}=\emptyset$ and hence $V_{l} \cap \widetilde{U_{m}}=\emptyset$ for $l \geq m+2$. Thus we see that if $k \geq 2$ and $z \in V_{k}$ so that $f^{n+1}(z) \in V_{k+n+1}$, then

$$
f^{n+1}(z) \notin \widetilde{U_{n+1}}=\widetilde{f^{n}\left(U_{1}\right)}
$$

Choosing $L:=1$ in Lemma 3 we see that $V_{k} \subset A(f)$ for $k \geq 2$. This completes the proof of Theorem 2 .

\section{REFERENCES}

[1] I. N. Baker, The domains of normality of an entire function. Ann. Acad. Sci. Fenn. Math. 1 (1975), 277-283.

[2] I. N. Baker, An entire function which has wandering domains. J. Australian Math. Soc. (Ser. A) 22 (1976), 173-176.

[3] I. N. Baker, Wandering domains in the iteration of entire functions. Proc. London Math. Soc. (3) 49 (1984), 563-576.

[4] I. N. Baker, Some entire functions with multiply connected wandering domains. Ergodic Theory Dynamical Systems 5 (1985), 163-169.

[5] W. Bergweiler, Iteration of meromorphic functions. Bull. Amer. Math. Soc. (N. S.) 29 (1993), 151-188.

[6] W. Bergweiler and A. Hinkkanen, On semiconjugation of entire functions. Math. Proc. Cambridge Philos. Soc. 126 (1999), 565-574.

[7] R. L. Devaney, Dynamics of entire maps. In Dynamical systems and ergodic theory, Banach Center Publications 23, Polish Scientific Publishers, Warsaw 1989, 221-228.

[8] L. Geyer, Quasikonforme Deformation in der Iterationstheorie. Diploma thesis, Technical University Berlin, 1994.

[9] M. Herman, Exemples de fractions rationelles ayant une orbite dense sur la sphère de Riemann. Bull. Soc. Math. France 112 (1984), 93-142.

[10] A. Hinkkanen, Uniformly quasiregular semigroups in two dimensions. Ann. Acad. Sci. Fenn. Math. 21 (1996), 205-222.

[11] M. Kisaka and M. Shishikura, On multiply connected wandering domains of entire functions. In Transcendental dynamics and complex analysis, edited by P. J. Rippon and G. M. Stallard, LMS Lecture Note Series, 348, Cambridge University Press, 2008, 217-250.

[12] C. T. McMullen, Complex dynamics and renormalization. Ann. of Math. Studies 135, Princeton Univ. Press, Princeton, NJ, 1994.

[13] P. J. Rippon and G. M. Stallard, On questions of Fatou and Eremenko. Proc. Amer. Math. Soc. 133 (2005), 1119-1126.

[14] D. Sullivan, Quasiconformal homeomorphisms and dynamics I. Solution of the Fatou-Julia problem on wandering domains. Ann. Math. 122 (1985), 401-418. 
Walter Bergweiler

Mathematisches Seminar,

Christian-Albrechts-Universität zu Kiel,

Ludewig-Meyn-Str. 4, D-24098 Kiel, Germany

E-mail: bergweiler@math.uni-kiel.de 